\title{
Presentación de "Continuum": el portal de formación continuada de la Asociación Española de Pediatría
}

Publicado en Internet: 10-diciembre-2013

Javier González de Dios javier.gonzalezdedios@gmail.com

\section{J. González de Dios ${ }^{\mathrm{a}}$, F. Hijano Bandera $\mathrm{F}^{\mathrm{b}}$, S. Málaga Guerrero $\mathrm{S}^{\mathrm{c}}$, Coordinadores de Continuum ${ }^{\mathrm{d}}$}

${ }^{a}$ Departamento de Pediatría. Hospital General Universitario de Alicante. España •bCS Monterrozas. Madrid. España • 'Área de Pediatría. Facultad de Medicina. Universidad de Oviedo. España

- dAlberto García Salido, Nuria García Sánchez, Manuel Molina Arias, Carlos Ochoa Sangrador, Javier Pérez-Lescure Picarzo, Manuel Praena Crespo, Carmen Villaizán Pérez.

\section{JUSTIFICACIÓN DE UNA PLATAFORMA DE FORMACIÓN CONTINUADA DE LA ASOCIACIÓN ESPAÑOLA DE PEDIATRİA}

Aprender y cambiar son dos elementos nucleares de la formación continuada, y son parte del objetivo de aprendizaje que deben realizar los pediatras para mantener su competencia profesional y prestar una óptima atención a la población infantojuvenil. El concepto del continuum, que ha estado clásicamente ligado a este objetivo, en la actualidad se relaciona más con la necesidad de diseñar estrategias para enlazar eficazmente la formación con la práctica médica.

Los profesionales demandan actividades no regladas de perfeccionamiento profesional, reciclaje y educación permanente. La formación a distancia, basada en el uso de las tecnologías de la información y las comunicaciones (TIC), es una forma de alcanzar este objetivo que posibilita y fomenta el aprendizaje interactivo, es flexible y accesible, y re- duce el aislamiento geográfico en el que se encuentran muchos profesionales.

Existen tres categorías de formación a distancia:

- Provisión de contenidos: materiales formativos para el estudio personal.

- Gestión de contenidos: reproduce el entorno de la formación tradicional, intentando recrear el aula: CD, vídeos, DVD, aulas virtuales.

- Formación en línea: parte de la idea de que el aprendizaje efectivo necesita diálogo, conversación, discusión y reflexión; el proceso formativo se concibe como un proceso social, no solitario.

La formación en línea (on-line) es una modalidad de enseñanza a distancia que usa Internet como canal de comunicación principal y utiliza múltiples recursos de las nuevas TIC. Se utilizan muchos sinónimos para expresar este medio de formación, con ciertos matices, como el de e-learning o webbased learning. 
A las ventajas ya comentadas, cabe añadir que esta modalidad de formación:

- Complementa la formación tradicional, con sus fortalezas y debilidades, con sus oportunidades y amenazas.

- Permite pasar de la información y formación estática (Web 1.0) a la información y formación dinámica, en la que se genera, comparte y modifica esta por parte de los usuarios (Web 2.0 o Web social). Hoy día no es posible concebir la formación continuada sin el recurso de Internet.

\section{CONTINUUM, EL PORTAL DE FORMACIÓN CONTINUADA DE LA ASOCIACIÓN ESPAÑOLA DE PEDIATRÍA}

Por todos los motivos previamente expuestos, nace un nuevo proyecto de la Asociación Española de Pediatría (AEP): Continuum, el Portal de Formación Continuada de la AEP. La AEP asume este proyecto porque cuenta con el liderazgo científico de sus 23 sociedades pediátricas de especialidades, sus 14 sociedades pediátricas regionales y sus 16 comités y grupos de trabajo.

Los objetivos de Continuum son:

- Generales: creación de un portal de formación dirigido a pediatras que tiene en común hablar el mismo idioma, en el que a través de diversas modalidades formativas (tanto individuales como grupales) se facilite la actualización de conocimientos, la más rápida integración de los avances médicos en la práctica y el perfeccionamiento profesional, que redunden en una mejor atención a la población infantojuvenil.

\section{- Específicos:}

1. Impartir una enseñanza innovadora, mediante planteamientos prácticos tipo "learning by doing", adaptando los contenidos a las metodologías docentes que hayan demostrado ser más eficaces para mejorar la competencia profesional.

2. Implicar a las subespecialidades pediátricas, grupos de trabajo y comités de la AEP en de- finir las áreas de conocimiento y competencias específicas que deben ser abordadas.

3. Recoger las necesidades de formación específica de los profesionales, así como los intereses estratégicos y/o institucionales, para planificar las actividades de formación.

4. Fomentar la mejora de la actividad asistencial, docente, científica e investigadora, monitorizar los resultados formativos, y recogerlos en un portfolio personal, que pueda servir de aval en un futuro para revalidar la capacitación en Pediatría.

5. Favorecer la obtención de créditos de formación continuada por actividad, que puedan ser aportados para la carrera profesional.

\section{¿QUUÉ PODEMOS ENCONTRAR EN CONTINUUM?}

Continuum es mucho más que una plataforma de cursos on-line. En ella, el usuario puede acceder a diversas modalidades formativas (algunas individuales y otras tutorizadas) y otros recursos de interés. Sus contenidos, distribuidos en 39 áreas temáticas (por ejemplo, Alergia, Bioética, Cardiología, Cirugía, Cuidados Intensivos, Dermatología, etc.), desarrollarán el Curriculum Global de Educación Pediátrica, una iniciativa impulsada por el Global Pediatric Education Consortium (GPEC) a la que se ha sumado la AEP. España será el primer país de la Unión Europea donde se ponga en marcha, así como el punto de partida para compartir el documento elaborado por el GPEC con los distintos países de Latinoamérica. Esta matriz de competencias es la hoja de ruta de Continuum y su principal valor añadido como portal de formación en línea.

Además, prácticamente todas las actividades dispondrán de la correspondiente acreditación por parte de la Comisión de Formación Continuada de las Profesiones Sanitarias de la Comunidad de Madrid, con validez en toda España.

En relación a las actividades de formación individual, Continuum dispone de cuatro secciones: 
- Imagen de la semana: es una sección en la que cada semana se publican imágenes relacionadas con enfermedades comunes de presentación poco frecuente, con objeto de ampliar la experiencia visual de los pediatras.

- Casos clínicos interactivos: de aparición bisemanal, su objetivo es promover el razonamiento clínico, incidiendo especialmente en el diagnóstico diferencialy la selección e interpretación de distintas pruebas complementarias, a partir de casos clínicos reales, tanto de Atención Primaria como de Atención Hospitalaria.

- Novedades bibliográficas: de aparición bisemanal, su objetivo es revisar las publicaciones más recientes (alrededor de 100 revistas biomédicas) con el objetivo de reseñar aquellos trabajos más innovadores o que puedan tener un interés especial, por el impacto clínico de sus resultados o por su contribución a la formación continuada de los médicos especialistas en Pediatría.

- Artículo destacado: de aparición bisemanal, enlazará con artículos publicados en las principales revistas pediátricas españolas (Acta Pediátrica Española, Anales de Pediatría, Anales de Pediatría Continuada, Cirugía Pediátrica, Evidencias en Pediatría, Formación Activa en Pediatría de Atención Primaria, Revista Española de Pediatría, Revista de Pediatría de Atención Primaria), que han dado su autorización para ello.

Las actividades tutorizadas son los cursos on-line, que ofertan periódicamente de forma preferente las distintas sociedades, grupos y comités de la AEP. Se plantearán cursos de diferente duración y créditos, que cubrirán las distintas áreas temáticas de la Pediatría, clínicas y no clínicas, contenidas en el Curriculum Global de Educación Pediátrica.

Otras herramientas de interés que Continuum pone a disposición de todos los usuarios son:

- Biblioteca: con la recopilación de documentos esenciales (guías de práctica clínica, revisiones sistemáticas, documentos de consenso, protocolos, artículos valorados críticamente) publicados en los últimos cinco años.
- Herramientas para la consulta: con múltiples recursos, tales como algoritmos diagnósticos y terapéuticos, calculadoras, consejos para padres y pacientes, tablas y valores de referencia, técnicas y procedimientos, vademécum, entre otras herramientas.

- Portfolio personal, llamado "Mi Continuum", donde los alumnos pueden recopilar las actividades realizadas y valorar sus necesidades de formación por áreas temáticas, según la matriz de competencias del GPEC.

- Agenda: para el anuncio de actividades de formación avaladas por la AEP y novedades de la plataforma.

- Twitter: como recurso útil de comunicación en el entorno 2.0.

\section{LAS FORTALEZAS DE CONTINUUM COMO PORTAL DE FORMACIÓN VIRTUAL}

Las señas de identidad de Continuum, y por tanto sus fortalezas, son las siguientes:

- Continuum es propiedad de la AEP, líder en formación pediátrica.

- La formación será impartida por expertos en cada competencia, en su mayoría miembros de los comités y los grupos de trabajo de la AEP, así como de las distintas sociedades de especialidad.

- Los temas a desarrollar son los contenidos en el Curriculum Global de Educación Pediátrica. También se abordarán las últimas novedades científicas para facilitar su integración en la práctica clínica.

- La metodología docente empleada promueve la interacción del alumno para la adquisición de habilidades concretas, y la tutorización de las actividades grupales.

- Los socios dispondrán de una sección, "Mi Continuum”, donde podrán valorar sus necesidades de formación, además de recopilar las actividades realizadas en Continuum, con los créditos de formación obtenidos. 
- Los contenidos estarán disponibles en cualquier momento y lugar. La plataforma ha sido diseñada para que su funcionalidad sea máxima en los dispositivos móviles.

- Es una plataforma de todos y para todos los socios, con la que la AEP pretende, de forma pionera en España, que estos mantengan su competencia profesional y sigan prestando una óptima atención a la población infantojuvenil.

Os damos la bienvenida a Continuum, el Portal de Formación Continuada de la AEP.

\section{CONFLICTO DE INTERESES}

Javier González de Dios y Francisco Hijano Bandera son codirectores de Continuum. Serafín Málaga Guerrero es Presidente de la AEP.

\section{ABREVIATURAS}

AEP: Asociación Española de Pediatría • GPEC: Global Pediatric Education Consortium - TIC: tecnologías de la información y las comunicaciones.

\section{BIBLIOGRAFİA RECOMENDADA}

- Asarbakhsh M, Sandars J. E-learning: the essential usability perspective. Clin Teach. 2013;10:47-50.

- Ellaway R. E-learning: is the revolution over? Med Teach. 2011;33:297-302.

- Feng JY, Chang YT, Chang HY, Erdley WS, Lin CH, Chang YJ. Systematic review of effectiveness of situated e-learning on medical and nursing education. Worldviews Evid Based Nurs. 2013;10:174-83.

- Frehywot S, Vovides Y, Talib Z, Mikhail N, Ross H, Wohltjen $\mathrm{H}$, et al. E-learning in medical education in resource constrained low- and middle-income countries. Hum Resour Health. 2013;11:4.

- Gordon M, Chandratilake M, Baker P. Improved junior paediatric prescribing skills after a short e-learning intervention: a randomised controlled trial. Arch Dis Child. 2011;96:1191-4.

- Grau-Perejoan O. Formación on line. Educ Med. 2008; 11:139-46.
- Khogali SE, Davies DA, Donnan PT, Gray A, Harden RM, MCDonald J, et al. Integration of e-learning resources into a medical school curriculum. Med Teach. 2011;33:311-8.

- Ruggeri K, Farrington C, Brayne C. A global model for effective use and evaluation of e-learning in health. Telemed J E Health. 2013;19:312-21.

- Sandars J, Lafferty N. Twelve Tips on usability testing to develop effective e-learning in medical education. Med Teach. 2010;32:956-60.

- Segura Badía H. Las nuevas tecnologías y la formación médica continuada a distancia. Educ Med. 2006; 9:118-26.

- Sharma N. The negatives of e-learning. Clin Teach. 2011;8:142-3.

- Thomson O'Brien MA, Freemantle N, Oxman AD, Wolf F, Davis DA, Herrin J. Continuing education meetings and workshops: Effects on professional practice and health care outcomes. Cochrane Database Syst Rev. 2001;(2):CD003030. 